\title{
Automation of the shoe last grading process according to international sizing systems
}

\author{
Juan José Hinojo-Pérez • Miguel Davia-Aracil • Antonio Jimeno-Morenilla • José Luis Sánchez-Romero • Faustino Salas
}

\begin{abstract}
The last is the basic industrial component in footwear manufacturing, from which product development starts. Correct last grading ensures the best fit for the intended group of users of the footwear model to be produced. The size marked on the last should respect the specific intervals defined in the different international sizing systems, like the European, UK, US or Mondopoint systems, which are all described in international standards. New approaches in the field of CAD/CAM have emerged over recent years towards the automation of this process. However, these are partial approaches that neither address the grading process according to the different standards nor consider the various parts of the shoe that are not affected by size increments. This paper presents a new accurate and efficient technique for the automation of the shoe last grading process based on the conjugate gradient method. Through this method, it is possible to obtain a graded shoe last that conforms to the international standards in force relative to shoe sizing and allows for the shoe parts that are not affected by size increments. This technique is based on the target measures of length and perimeter of the last to be graded, and aims to minimise the quadratic difference between these values and those obtained from the graded last. This method has been evaluated through a battery of tests performed on a geometrically heterogeneous group of shoe lasts. The results obtained were accurate and the execution time was fast enough to be used for mass production.
\end{abstract}

Keywords Footwear manufacturing • Grading • Shoe last • Conjugate gradient $\bullet$ ISO Standards

\section{Highlights}

- This paper presents an accurate and efficient technique for shoe last grading based on the conjugate gradient method.

- This paper describes a grading algorithm based on the minimisation of the error obtained from comparing measures between shoe lasts.

- This grading technique conforms to ISO standards relative to sizing systems and allows for footwear parts that are not affected by the grading process.

- This paper presents a detailed battery of experiments by which the goodness and accuracy of this method were evaluated.

\section{Introduction}

Traditionally, footwear manufacturing has been a handcrafted process that, even though products perfectly fit for consumer's feet were obtained, was expensive and inefficient. Industrial footwear manufacturing allows the manufacturer to maximise profits while high quality products are obtained. Shoe lasts are the basis for industrial footwear manufacturing [1], which is used as a physical model for the mass production of shoes.

Last design is a complex process that should consider foot morphological features, such as length and width. Foot dimensions differ from one individual to another, although it is possible to put them into groups of very similar dimensions. This is where sizing systems stem from $[2,3]$, which define a range of length and width measures based on several foot features. The industry usually produces a wide range of sizes of the same shoe model, requiring a different last for each size. The fastest way to obtain such lasts is by grading a base last to obtain bigger and smaller ones. This process is known as shoe last grading.

In a global market, the conformance of the shoe last grading process to ISO (International Organization for Standardization) standards relative to footwear sizing becomes essential. This is even more patent in the online sale of shoes, where the size marked on the shoe model needs to precisely match the buyer's foot length.
J. J. Hinojo-Pérez ${ }^{1}$ M. Davia-Aracil ${ }^{1,2}$.

A. Jimeno-Morenilla ${ }^{2 *}$ • J.L. Sánchez-Romero ${ }^{2}$

${ }^{1}$ Spanish Footwear Technology Institute (INESCOP),

${ }^{2}$ Department of Computer Technology, University of Alicante *Corresponding author data:

Postal address: Ctra. San Vicente del Raspeig S/N, 03690, Spain Phone: +34 965903400 Ext. 2453

Fax: +34965909643

e-mail: jimeno@dtic.ua.es 
The technical standardisation committee working on these standards is ISO/TC 137 - "Footwear sizing designations and marking systems". This committee works on the standardisation of the different sizing systems (European, Mondopoint, UK, US) so that a given size in a system can be converted into the corresponding size in another system. Likewise, the terminology and vocabulary used in this field related to sizing and foot and last dimensions, are also defined.

The manual grading of the base size last is a costly and inaccurate process. This is why the size range obtained tends to be reduced so as to be able to minimise costs, which means that a shoe in a given size may not perfectly fit the user's foot. The traditional mechanical grading process uses a copying lathe, where the two lathe dimensions, i.e. length and tool proximity, are modified accordingly. Given that the latter dimension is established by polar coordinates, too large grading causes corner rounding, so it is necessary to produce a new master for every 3 sizes. Furthermore, feet do not grow linearly in all their dimensions, since the ratio between foot length and ball girth increase is really small [4]. Therefore, accurate techniques are required for the automatic generation of the various sizes starting from a base last and taking into account the morphological features of feet. The latest CAD/CAM advances have led to different shoe last grading, design $[4,5,6,7,8]$ and manufacturing approaches starting from the designed models.

This paper aims to propose a new method for the automated grading of shoe lasts based on the conjugate gradient technique, which allows a graded last to be quickly and accurately obtained. To ensure adequate fit, the grading procedure will observe the intervals established in the different international shoe sizing systems described in international standards.

The paper is structured as follows: Section 2 firstly introduces the main foot features and their extrapolation to last dimensions; then the main sizing systems are presented; and finally there is a critical review of the main contributions to automatic last grading by means of computing tools. Section 3 defines the fundamentals of the conjugate gradient grading technique. Section 4 provides a detailed description of the proposed procedure; firstly, the pre-requirements for the application of the technique are explained; then the grading algorithm is presented. Section 5 describes the experiments carried out to check the validity of the method in terms of efficiency and accuracy, drawing the conclusions from the obtained results.

\section{State of the Art}

The evolution of CAD/CAM techniques has resulted in the application of computing tools for last design, which allow the development and processing of the last in 2D and 3D. Software applications have also been implemented for last grading and obtaining the different sizes [5,9], making this process more accurate and efficient. Natural foot growth and hence size grading are very complex processes. There is great variability in foot morphology, which leads to a big number of sizes. And furthermore, there are different sizing systems all over the world, which makes process automation even more complicated. Some of these systems only take foot length into account for the designation of sizes in order to reduce costs.

\subsection{Foot features and last dimensions}

There are certain measures and feature points of the foot which are essential to determine its morphology. These measures were characterized in [10]. Additionally, a correspondence analysis between the foot and the shoe last was carried out in order to determine how these two geometries must grow. The increment or decrement of such measures will result in different lasts, which in turn will allow the production of different shoe sizes.

One of the most commonly used measures is foot length, i.e. the horizontal distance from the foot tip (most prominent toe) to the rearmost point of the heel. There are also girth measures, the girth being a line encircling a cross section of the foot at a specific point. The three girth measures are: ball girth, waist girth and instep girth. Heel height and toe height are also important measures. Finally, bottom width is the straight-line distance between the lateral waist and the medial waist, i.e. it is a foot breadth measure. Figure 1 shows these measures.

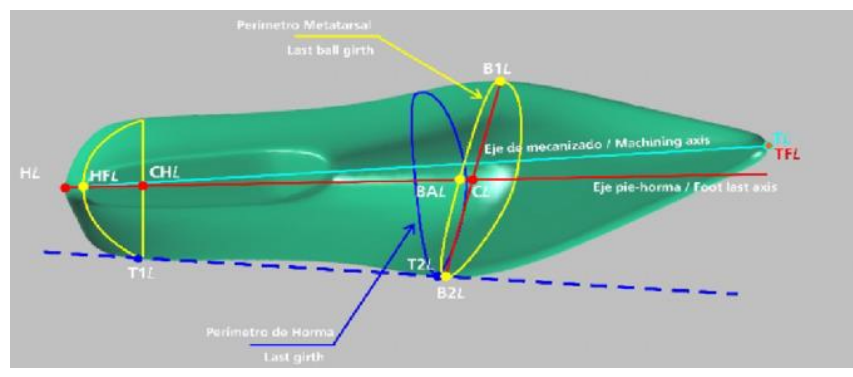

Fig. 1 The last and its main dimensions.

For mass footwear production, manufacturers usually put feet into groups according to their dimensions. These groups are known as 'sizes'. Traditionally, the most frequently used measures for the determination of sizes were foot length and ball girth. However, different studies [4] have demonstrated that the increase in foot length and ball girth is not proportional. Furthermore, girth measures (ball, waist and instep) are largely correlated to each other and to other measures, except for foot length. In addition, ball girth is the only girth measure that cannot be adjusted by the shoe fastening. This way, the measures that better allow for foot growth are foot length and ball girth.

\subsection{Sizing systems}

Footwear manufacturers and standardisation bodies have developed sizing systems [2,3,5] that can be classified into two categories. Firstly, there are systems based on the 
effective last length, i.e. the last the best fits a foot of certain dimensions, with which a shoe of a given size designated by a number (e.g. 42 or $4 \frac{1}{2}$ ) is produced. Some examples of this category are the European or Paris Point system (used in many European countries), the US and the UK systems. The second category includes systems based on the foot length, as is the case of the Japanese or the Chinese systems.

These systems are usually based on foot length and ball girth measures to designate sizes. A unit of measure (inches for the UK system or mm for the European system) is established and a length unit representing the intervals between sizes is defined (e.g. $6.6 \mathrm{~mm}$ in the European system or $1 / 3$ inches in the UK system). The starting point for some systems is 0 (European system), while others start at a different point (100 $\mathrm{mm}$ in the US system, 4 inches in the UK system). The ball girth interval for each length unit is determined (e.g. in the European system, $5 \mathrm{~mm}$ is the length interval and $1.67 \mathrm{~mm}$ is the bottom width interval). These measures can vary according to the consumer's gender and age.

In general, sizes designate foot length, gender and age, although the increase in foot length is not closely correlated with other measures, such as ball girth. Other systems, such as the Japanese system, also define sizes based on the ball girth, in addition to the sizes based on length. Thus, size E corresponds to an average women's ball girth measure, which can turn into EE, EEE and EEEE as the ball girth increases, or into $\mathrm{D}, \mathrm{C}, \mathrm{B}, \mathrm{A}$, as the ball girth decreases. A Japanese size will be expressed as the foot length in $\mathrm{cm}$ followed by the ball girth size.

The Technical Committee ISO/TC 137 is in charge of harmonising international sizing systems by defining both a set of technical specifications relative to foot and last measurement. These measures will be key to the designation of the shoe size for a reference foot, and a set of conversion tables between the existing sizing systems, so that users can calculate their sizes, no matter their origin country or the sizing system used for marking the shoes to be purchased [11,12]. The adequate way to obtain the length of a last suitable for a reference foot does not consist in measuring the distance between the frontmost and the rearmost points of the last. In fact, the geometry of the last toe can largely vary from one last to another due to fashionrelated design issues, which have nothing to do with biomechanical aspects of the foot. The standards establish that size designation should be based on the effective last length, which is directly related to foot length. This measure is based on foot length plus a toe allowance to adequately accommodate the foot inside the shoe to perfectly perform its biomechanical functions. In Spain, the toe allowance is usually $5 \%$ of the foot length (adults' shoes; $8 \%$ for children's shoes). This way of establishing the shoe size ensures adequate fit regardless of the last toe design.

\subsection{Shoe last grading techniques}

There have been several proposals in recent years to address the automatic last grading process. Among them, there are systems that provide information to generate sizes according to certain morphological features of the foot [1,2] and others that automatically generate the last from a reference template $[4,5,6,7,8,9]$ obtained by using a CAD tool or by $2 \mathrm{D}$ or $3 \mathrm{D}$ scanning.

Several studies introduce comprehensive software tools for last grading. Driscu, and Costea [5] proposed a tool called Delcam Crispin 3D - Last Maker. The grading function is a part of the tool, which requires certain 3D last parameters to be adjusted: toe spring, heel height, last length and ball girth. The tool allows last grading to be automatically performed for more than one size at once, and also allows the selection among different sizing systems and, within them, the choice of gender/age, length and width of the target size. Once the last grading file is obtained, the last can be manufactured using whatever of the existing CAD/CAM approaches [13,14]. This approach did not describe in detail the equations employed to grade the last, but highlighted that the grading is performed proportionally. As mentioned above, several studies concluded that there is little correlation between length increase and ball girth increase[4], therefore, it would be necessary to analyse the results of the proposed method to check the adaptation of the resulting last to the user's foot.

Luximon, Zhang and Xiao [7] proposed a more comprehensive method than the previous one, which is based on the principal component analysis (PCA) on 3D surfaces for the automatic grading of wearable products and, hence, the creation of a statistical grading model. In the case of shoe lasts, the feet of 60 individuals were scanned, in such a way that each foot was represented by a point cloud. The points were filtered and aligned by means of an algorithm based on geometric information, and from them a region of interest (RoI) was defined, which should match for the lasts for all feet analysed. Then, sampling was performed to compare the number of points and their order in each sample. Once the samples had the same number of points, the PCA algorithm was applied to reduce the dimensionality of a data set in which there was a large number of interrelated variables, while maintaining as much as possible the variation present in the data set. The grading rule was determined based on linear equations defined according to the correlation between geometric points and the morphological variables of the foot, such as foot length or flare angle. Based on such correlation, the regression equations were obtained, which allowed the grading to be performed based on these features. This procedure is depicted in figure 2. The average error obtained using this method was $2.03 \mathrm{~mm}$. for the left foot and $2.09 \mathrm{~mm}$. for the right foot; whereas using a proportional grading technique, it was $2.68 \mathrm{~mm}$ for the left foot and $2.75 \mathrm{~mm}$ for the right foot. Even though the results 
were improved, there was still a loss of accuracy that makes the method inadequate.

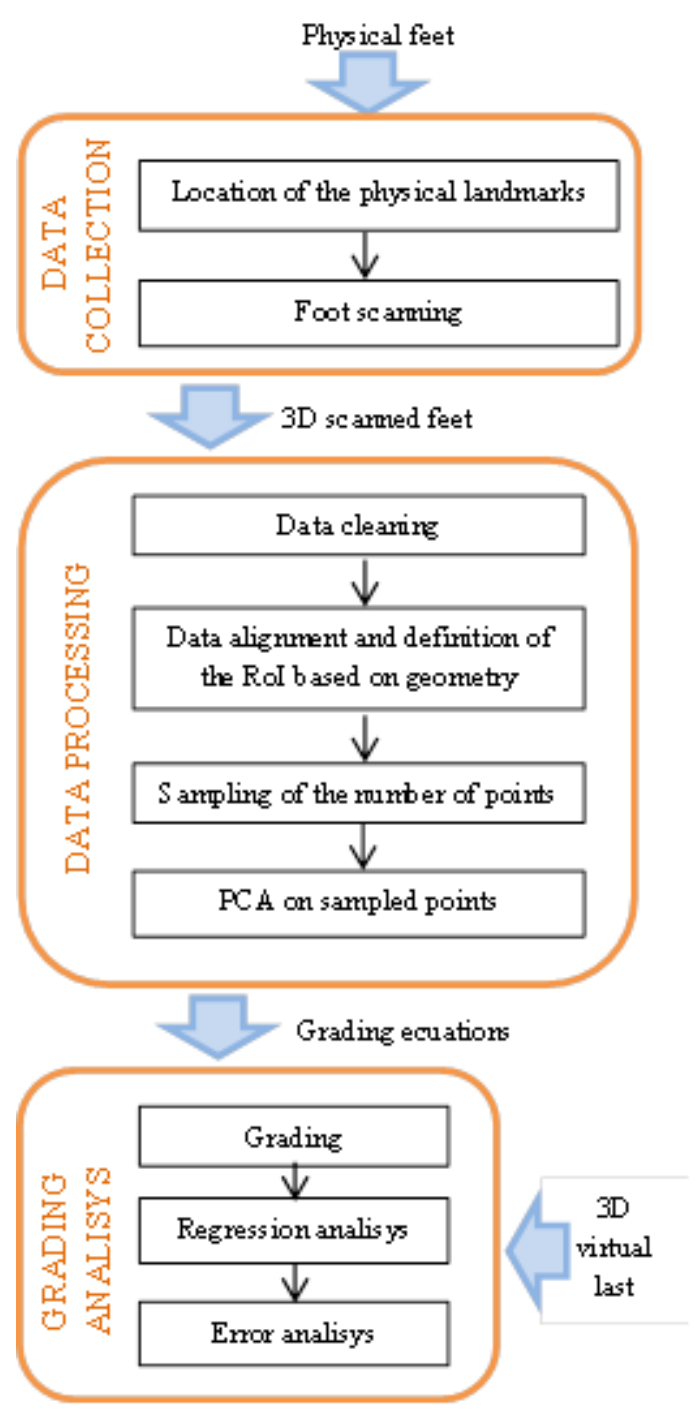

Fig 2. Last grading method based on PCA.

Other approaches $[4,6,8]$ were based on the deformation of 3D surfaces. Xiong, Zhao, Jiang and Dong [8] presented a system to créate a customized shoe last based on the morphologic features of the customer's foot and a chosen shoe style gathered from a base last. Shoe last grading is an essential operation inside this customization procedure, which is graphically described in figure 3. Firstly, the customer's foot is scanned and a cloud of 90,000 3D points approximately is obtained. The next step consists on the extraction of 18 features (five lengths, four widths, three heights, six girths) that will allow the foot characterization. In order to ensure the consistency of these measures, an automatic alignment is carried out through an algorithm to adjust the foot orientation. An uniform grading is applied on the base shoe last after the measures have been characterized. This grading is calculated through the minimisation of the weighted differences between the ideal design parameters and shoe last parameters after scaling. The weight assigned to each parameter depends on the importance for foot shoe fit. Finally, the user applies a number of deformations manually to adapt the shoe last to the foot in all its regions. Although this grading method would be appropriate for individuals as long as the uniform grading is corrected by the deformations that are applied to the last, it is not valid for mass production. This is because the last is adapted to a particular foot, and not to a target size that would be appropriate to meet the fitting requirements of a wide range of customers. These requirements change depending on the geographic region to which the product will be addressed, hence time-to-market would become a critical restriction if this method was integrated in footwear production.

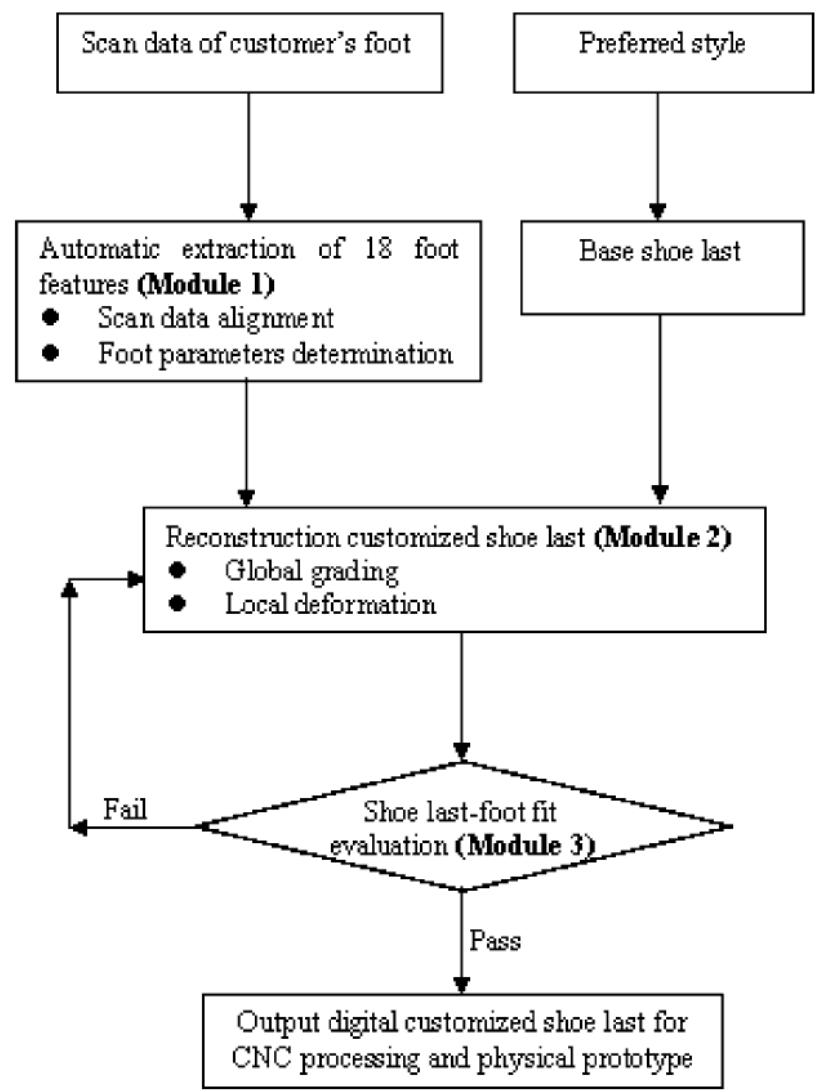

Fig 3. Customization procedure described in [8].

Mochimaru, Kouchi, and Dohi [4] proposed a method for the analysis of foot shape based on the FFD (free form deformation) technique. This technique is a way to deform a 3D object smoothly by moving control lattice points set around the object as shown in figure 4, and allows the measurement of dissimilarities between sizes. In this case, it was based on identifying the morphological features that mostly affect the changes in last shape. For this, 56 feet were digitised and 174 coinciding control points were defined. To obtain the displacement formula, an equation consisting of two members was proposed. The first member represents the sum of the differences between the original control point lattice and the modified lattice. The second member represents the sum of the differences between the control points of the original object and those of the target object. The result is multiplied by a $\mathrm{W}$ factor. The 
difference between the original object and the target object is defined as the sum of the Euclidean distance of the control lattice movements. In [4] an experiment based on the Japanese system was conducted, which consisted in comparing the grading of a $23.5 \mathrm{E}$ shoe last to a much wider last, size 23.5 EEEE, by means of the conventional method and by the FFD method. It was observed that the last obtained with the FFD method was narrower at the heel and wider at the ball girth and toes than the last obtained with the conventional method. Likewise, the thickness at the toes was not so large with the FFD method. Therefore, the proposed method reflected the allometric differences between narrow and wide feet better than linear grading methods, as is the case of the conventional method or the one proposed in [5].However, [Kim, Shin and Chung [6] claimed that the FFD based methods, as the one in [4], are not suitable for grading, in that it is very difficult to exactly calculate the control points required for the accurate deformation of the original shape to obtain the target shape. In addition, the computational cost and the storage required are too high.

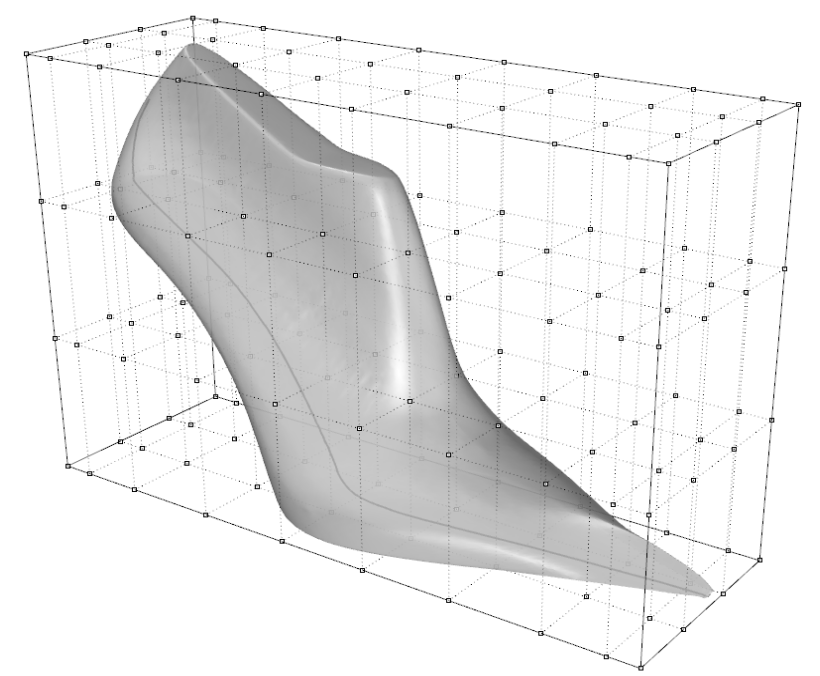

Fig 4. Point mesh used for grading through FFD. The mesh cannot be adapted to the last geometry, hence there is little control for applying grading in a correct way.

Kim, Shin and Chung proposed a method [6] also based on the deformation of a $3 \mathrm{D}$ surface, which is applicable to last grading as well as shoe upper and outsole grading. This method allows deformed surfaces to be constructed from the original surfaces on which a set of feature points and displacement vectors had been defined. Such vectors represent the constraints that define the deformation of the original surface. Unlike the method described in [4], this new method combines FFD and the extension of the 2D MBA (Multi-Level B-Spline Approximation) algorithm for the deformation of 3D surfaces. The study concluded that it is critical to devise the initial and boundary conditions of the deformed surface. Contrary to [4], it does not mention how a last is graded from one size to another in a specific sizing system. The case study here only defines a displacement vector field for grading one last and its initial and boundary conditions. In one of the tests, the execution times for generating a vector field and grading are calculated, obtaining 4.5 and 276 s. respectively. Due to the excessive execution time, this method would not be suitable for the footwear industry.

Finally, it should be stressed that, regardless of the accuracy of the techniques analysed above, none of them follows the guidelines given by the international standard in relation to the suitable way of establishing last size according to the effective last length; therefore, the lasts obtained by means of these systems would not comply with the international standard [11,12]. The usage of effective length as a reference measure for last grading is essential for its proper correlation with the foot growth.

\section{Conjugated gradient method for grading}

This method aims to minimise the quadratic error of the difference between the measures of the last to be graded and the target measures. The reference measures will be the effective length and the ball girth. For a base size last, being aware of the fact the increments between sizes are not proportional, it is necessary to find the parameters that allow it to be graded and match specific effective length and ball girth measures. This method will be generic and may be used with women's, men's and children's lasts, regardless of the heel height. Through this method, it will be possible to obtain, in a quick and accurate way, the whole range of sizes required for the production of a footwear model, while complying with the international standard relative to shoe sizing.

In order to carry out the grading process, it is necessary to determine the constant parameters by which the geometric coordinates of the vertices making up the discretised geometry will be multiplied. This way, the proportion will be adapted to the desired one. There will be two parameters to be applied in the form of a grading matrix. On the one hand, the length factor, expressed as $\alpha$, and on the other hand, the width and height factors, which will be grouped together and expressed as $\beta$. The fact that the last geometry that has been graded based on these factors is erroneous when compared with the desired last is a problem to be minimised and solved by means of minimum values of quadratic functions between two entities of similar but not identical geometry. Moreover, the error derived from the discretisation of a surface shall also be taken into account.

The problem can be formally described as follows: Let $\{\mathrm{V}\}$ be the set of vertices making up the mesh of the virtual last and $\left\{\mathrm{V}^{\prime}\right\}$ be the set of vertices making up the mesh of the graded virtual last.

The ScaleLast function defined in expression (1) changes the position of last vertices to adapt it to its new dimensions 
ScaleLast $:\{V\}, \alpha, \beta \rightarrow\left\{V^{\prime}\right\}$

ScaleLast $(\{V\}, \alpha, \beta)=v_{i}{ }^{\prime}=v_{i} \cdot T$

$\forall v_{i} \in\{V\} \forall v_{i}^{\prime} \in\left\{V^{\prime}\right\}$

Where the grading transformation is defined according to expression (2).

$$
T=\left(\begin{array}{ccc}
\alpha & 0 & 0 \\
0 & \beta & 0 \\
0 & 0 & \beta
\end{array}\right)
$$

The energy or target function, as shown in expression (3), consists in minimising the quadratic difference between the effective length and the ball girth desired in the graded last and those obtained by searching the closest grading parameters $\alpha$ and $\beta$ :

$$
\text { Min }=(\text { ActualLength }- \text { TargetPerimeter })^{2}+
$$

\section{(ActualPerimeter) - TargetPerimeter) ${ }^{2}$}

Where ActualLength and ActualPerimeter are the length and ball girth of the graded last under evaluation, and result from the application of the procedures GetLength and GetPerimeter, respectively. These serve as the basis for comparison and error finding between lasts. This way, if the result is 0 , this means that the effective length and ball girth of the graded last totally match the desired measures. The GetPerimeter procedure obtains the ball girth automatically. However, the GetLength procedure uses the start point and end point of the effective length, which will be set by the designer on the digital last based on their experience.

The reference measure for the calculation of the shoe size is the effective last length. According to the international standard, the effective last length is defined as the foot length value plus toe allowance, as indicated in expression (4). In Spain (and also in this paper) toe allowance is defined as $5 \%$ of foot length (adults' footwear).

\section{EffectiveShoeLength $=$ FootLength + ToeAllowance}

The international sizing systems considered in this study are the European (Paris Point), the UK and MondoPoint systems. In each system, size is defined according to expression (5).

SizeParis = EffectiveShoeLength $/ 6.66$

SizeUK = EffectiveShoeLength $/ 8.47-25$

SizeMP $=\operatorname{trunc}($ EffectiveShoeLength, \pm 5$)$

\section{Algorithm description}

\subsection{Previous steps:}

a. Discretisation of triangular mesh surface: In order to first carry out the comparison between lasts, which is the basis of the proposed method, and then grade the last in an efficient way, the surface defining the original last needs to be discretised. This would be required when dealing with a continuous surface, e.g. a NURBS surface. Additionally, a point cloud obtained from the digitisation of the original physical last could be used. In this case, the discretisation would be implicitly provided by the digitiser resolution. Through this process, a triangle mesh is obtained for which the vertices position will be the basis for grading, since grading is performed from their modification. This way, the graded mesh will have the same number of triangles as the original one, but their areas will be smaller or bigger depending on whether it is graded to a bigger or smaller size. With a view to minimising errors, it is recommended to work with meshes in which triangles have similar areas.

b. Identification of effective length points. The user, an expert last designer, shall establish the start and end points of the effective 3D last length. This way, it is possible to determine the length directly related to the foot morphology on which grading is based, and to neglect the irrelevant part of the last that is exclusively dependent on fashion criteria.

c. Surface positioning. In order to apply this grading method on the virtual last, the last needs to be in a specific position in the coordinates system. In this position, which is illustrated in figure 5, the machining axis of the last (longitudinal heel-toe axis) shall coincide with the $\mathrm{X}$ axis. This means that the heel or rearmost point shall be positioned at $\mathrm{X}=0.0 \mathrm{~mm}$. The last shall be centred with respect to the $\mathrm{Y}=0 \mathrm{~mm}$. plane, in such a way that it is split into two halves. The cone top shall be positioned in the direction indicated by the $\mathrm{Z}$ axis.

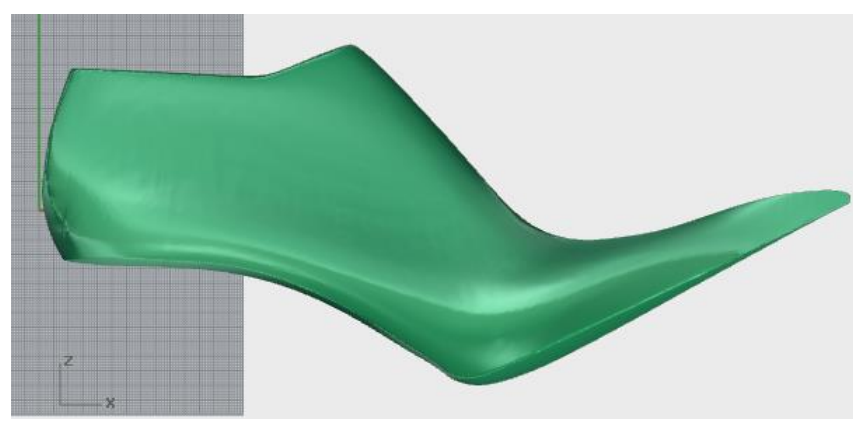

Fig. 5 Virtual last view on the XZ plane, adequately positioned for the application of the grading method.

\subsection{Algorithm:}

The proposed algorithm is based on the optimisation of the energy function, so as to minimise the difference 
between the effective length and the ball girth of the graded last and the reference values to be graded. This is achieved by applying the conjugate gradient method. The choice of the adequate values to initialise the variables allows a valid solution to be found after a reduced number of iterations.

The energy function is minimised by means of the gradient method, since the problem to be solved consists of a nonlinear equation with two unknown factors.

The variables to be used for length grading $(\alpha)$ and width and height grading $(\beta)$ shall be initialised with values close to the solution provided by accurate grading, so that the method converges with the least number of iterations possible. The approximate solution, with which the variables will be initialised, will be obtained from the calculation of the proportions in which the effective length and the ball girth will be altered, as indicated by the expression (6).

$\alpha_{\text {init }}:=$ TargetLength/OriginalLength

$\beta_{\text {init }}:=$ TargetPerimeter/OriginalPerimeter

Once the initial values have been calculated, the grading algorithm is applied. Using the conjugate gradient method, the algorithm is based on the minimisation of the energy function that calculates the difference between the desired length and ball girth and the obtained ones.

The method outputs are the $\alpha$ and $\beta$ variables making up the grading matrix, as defined in expression (7):

$X=(\alpha, \beta)$

Where the residual error is:

Error $=(\text { ActualLength }- \text { TargetLength })^{2}+$ (ActualPerimeter - TargetPerimeter $)^{2}$

The ActualLength and ActualPerimeter variables in expression (8) are equal to the result obtained from the application of the GetLength and GetPerimeter respectively on the current graded geometry. These procedures serve as the basis for the comparison of lasts in that they allow the quantification of the geometric difference between the graded sizes of the same last. With each method iteration, the last is graded and the error is calculated by these two methods.

Given that there are restrictions in the range of values that the output variables may have, such cases will be identified and punished by assigning a high error value without the need of comparing the lasts. The restrictions to be met are defined in expression (9):

TargetLength $>$ BaseLength $\rightarrow \alpha>1$

TargetLength $<$ BaseLength $\rightarrow \alpha<1$

TargetPerimeter $>$ BasePerimeter $\rightarrow \beta>1$

TargetPerimeter $<$ BasePerimeter $\rightarrow \beta<1$

\section{GetLength Procedure:}

The effective last length is determined by obtaining the distance on the last surface between the minimum and maximum points of its effective length on the $\mathrm{X}$ axis, as shown in figure 6 . That is, the points on the last corresponding to the minimum and maximum points on the $\mathrm{X}$ axis of the foot for which the last has been designed. The start and end points defining the effective last length are to be entered as parameters, since their automatic calculation is not a trivial issue and should be carried out manually by a designer.

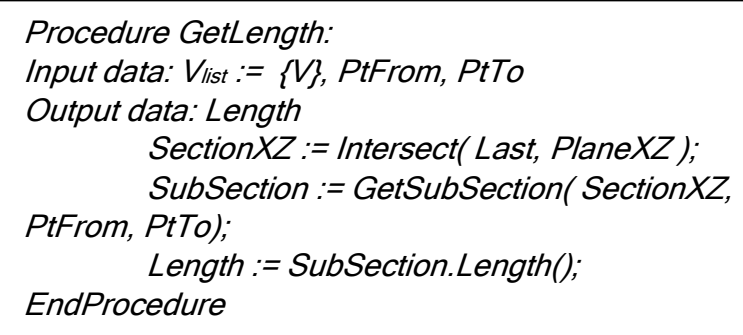

Fig 6. Procedure for the calculation of the effective last length.

GetPerimeter Procedure:

The ball girth of the virtual last is determined from the ball points, as illustrated in figure 7 . These are characteristic points on the last surface delimiting the geometry of the metatarsal area. There are three points: the inner point, the outer point and the central point. Starting from the position defined in previous sections, the BallInner point and the BallOuter point correspond to the most prominent vertices on the right and on the left of the foot (minimum and maximum $\mathrm{Y}$ points of the bounding box). The BallCenter point is the point within the cross-section of the last resulting from the intersection with the $\mathrm{XZ}$ plane closest to the inner and outer points. Figure 8 describes the procedure for calculation. From the BallInner, BallOuter and BallCenter points, a plane is created with which the last is intersected. The length of the resulting cross-section will be the last width.

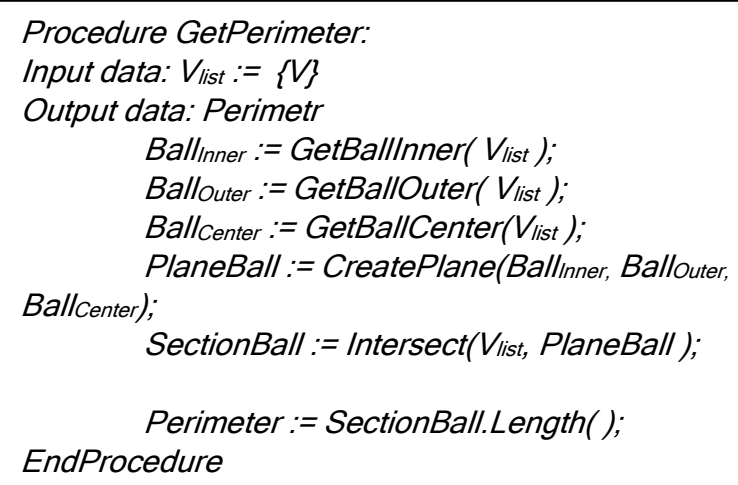

Fig 7. Procedure for the calculation of the last ball girth. 


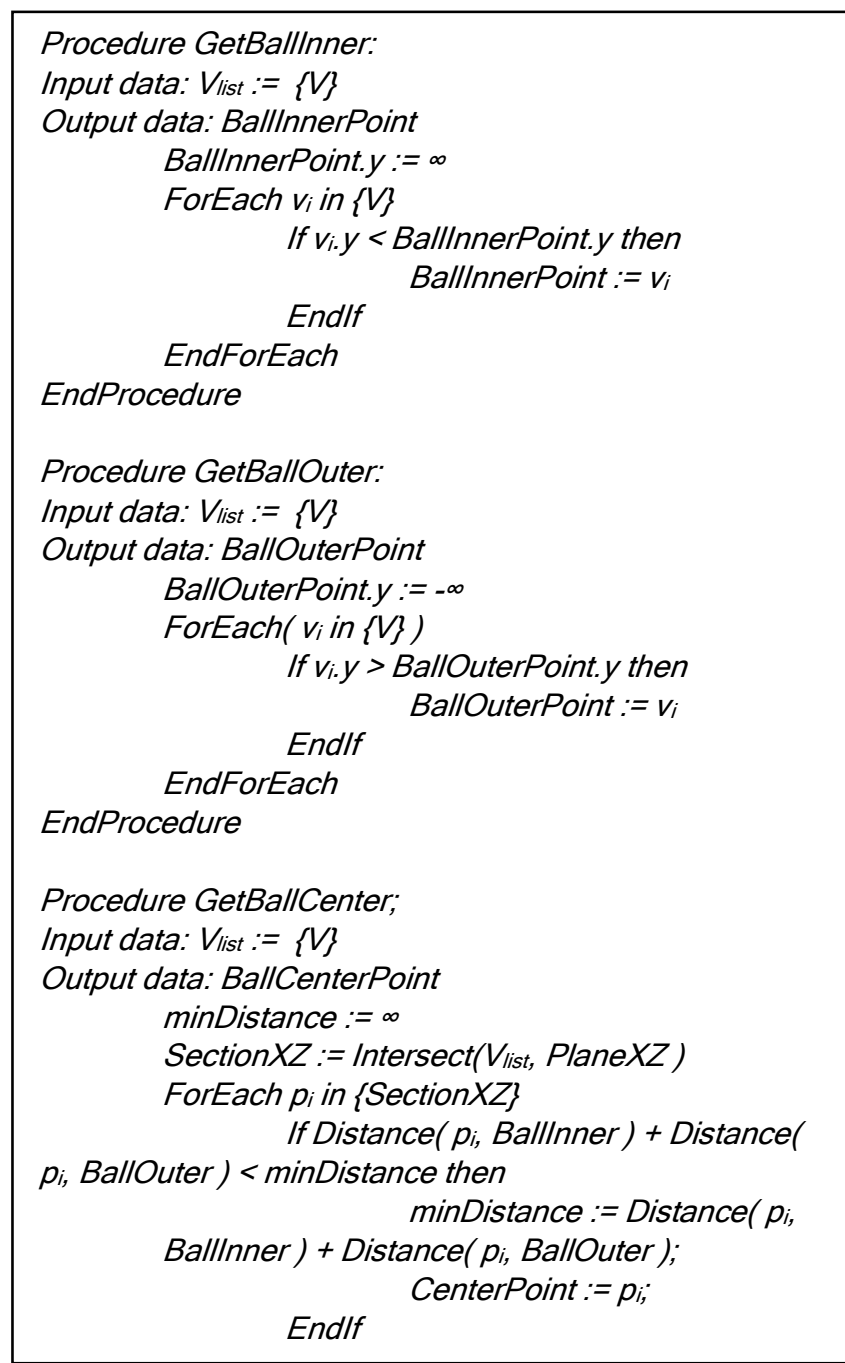

Fig8. Procedure for obtaining the ball points required for girth calculation.

\section{Experimental}

The experimental stage was aimed to check firstly the robustness of the proposed method, so that the error of the grading operation is minimal; secondly, its genericity, i.e. the method should be generic enough to be able to be applied to any type of last; and finally, it was also checked that the execution time was reduced. For this, four lasts were chosen, which can be considered representative within the spectrum of morphologies used in the footwear sector and on which all the experiments were carried out. Table 1 shows the names assigned to each last and their typologies.

\begin{tabular}{l|l}
\hline Name & Type of last \\
\hline Last1 & Women's last with high heel height \\
\hline Last2 & Women's last with medium heel height \\
\hline Last3 & Women's last with low heel height \\
\hline Last4 & Men's last \\
\hline
\end{tabular}

Table 1: Name and description of selected lasts

The selected lasts, which are depicted in figure 9, were provided as a NURBS surface. To be able to work with them, each one was discretised into a triangle mesh in three different resolutions ( $\mathrm{H}, \mathrm{M}$ and $\mathrm{L})$. The $\mathrm{H}$ (High) resolution discretisation is based on the parameters generated by a mesh of triangles of variable sizes according to the original surface, which in the case of these lasts ranged from 41,000 to 100,000 vertices. $\mathrm{M}$ and $\mathrm{L}$ resolutions corresponded to meshes with a fix number of vertices, i.e. 30,000 and 10,000 respectively. The objective of working with the same geometry in different resolutions was to evaluate the execution speed of the algorithm as the number of discretisation triangles decreased while comparing the measures obtained in different instances. This way, it would be possible to determine if the error derived from the definition loss is acceptable and, therefore, allows grading parameters to be obtained, which are also valid for the original geometry.

Starting from a last in a given base size and using the Paris Point sizing system, the experiments consisted in grading by increasing and decreasing two sizes in ball girth and length, with an average of half size intervals between sizes. For each last model and surface discretisation resolution, this operation resulted in a new series of 8 triangle meshes (figure 10). Among them, 4 are smaller lasts than the original one, while the other 4 are bigger (table 2). For each of the results obtained, the error derived from the grading operation and the time taken was assessed. 

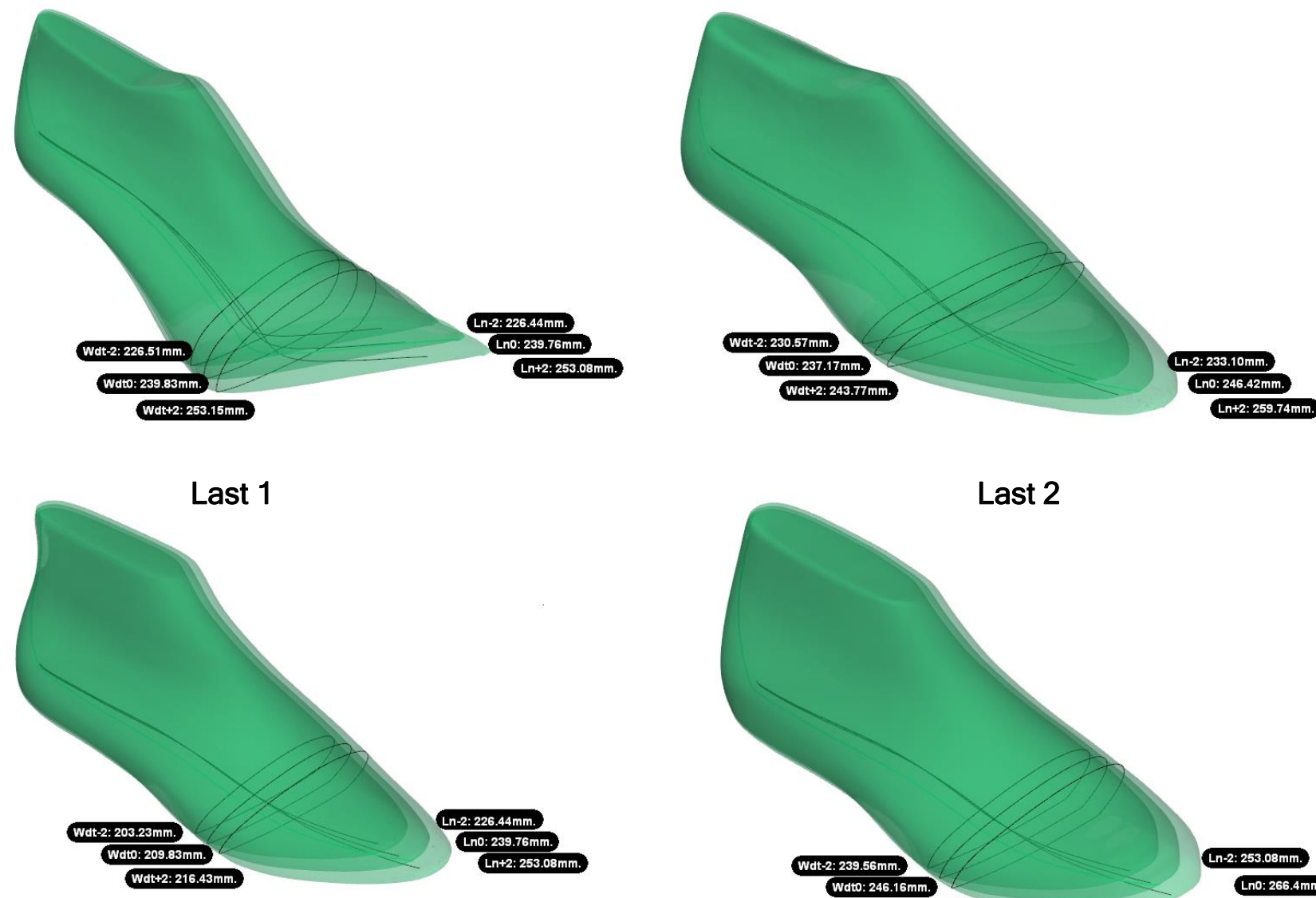

Last 3

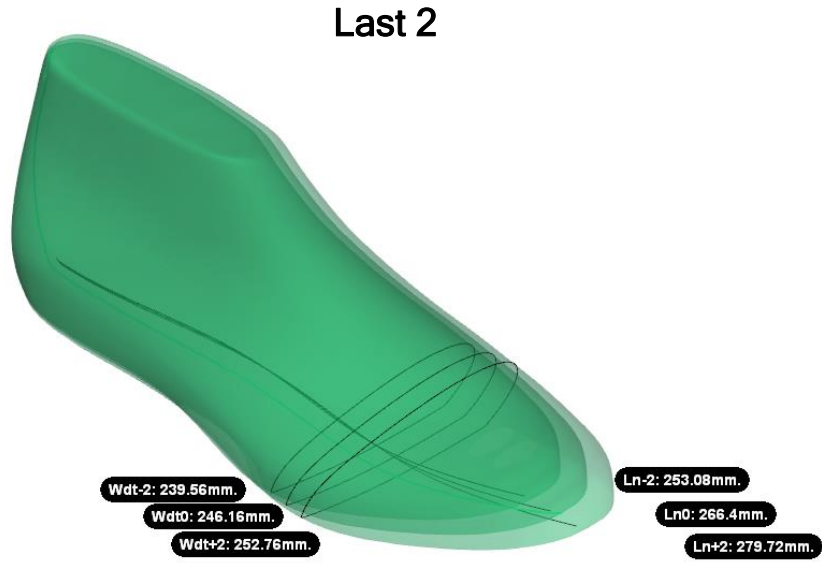

Last 4

Fig. 9 Original lasts and lasts graded by -2 and +2 together with the curves defining their effective length and ball girth.

This experiment was carried out on a computer with the following features: 8-core, $2.67 \mathrm{Ghz}$ Intel Core i7 920 processor, 6 GB RAM memory and Windows 7 OS.

In all the tests carried out, the error resulting from the adjustment of square minimums was equal to 0 . This means that the achieved geometry fully matched the desired one, according to the results provided by the effective length and ball girth functions of our method. However, it is advisable to determine the error derived from working with meshes discretised at different resolutions with respect to the original surface. This is due to the fact that when working at lower resolutions, even though the last is graded in less time, there is a loss of information about the geometry. It should be determined whether this factor decisively affects the accuracy of the results

The error was quantified by comparing the measures obtained from the meshes discretised at $\mathrm{H}, \mathrm{M}$ and $\mathrm{L}$ resolutions with the result obtained by grading the original surface. However, the original surface is not a discrete model and, hence, cannot be graded with the proposed method. With the aim of using a mesh that is as approximate as possible to the original surface geometry, it was discretised at a much denser resolution. This means working with meshes of over 500,000 polygons per model, for which the proposed method could not be directly applied, since the execution time taken would render it unfeasible and unpractical.

The effective length and ball girth errors analysed below consist of the difference in $\mathrm{mm}$. between the last measures graded at $\mathrm{H}, \mathrm{M}$ and $\mathrm{L}$ resolution, and the measures obtained from the ideal last after the application of the respective $\alpha$ y $\beta$ parameters resulting from the grading of the trio of meshes at a lower resolution.

- Effective length calculation error: In all experiments, the error obtained when comparing the effective length calculated with the optimal resolution mesh was less than $0.1 \mathrm{~mm}$. As shown in table 3, in general, the error increased as the resolution decreased. Nevertheless, since this value was in no case significant, we will not go into detail about it.

- Ball girth error: Unlike the previous case, the ball girth error values were significant and should be analysed. Roughly speaking, we observed that the error values increased as the resolution of the graded geometry 
decreased (table 4). When looking at the maximum error values, we found that for $\mathrm{H}$ res the value was $\pm 0.46 \mathrm{~mm}$, for $\mathrm{M}$ res. $\pm 0.57 \mathrm{~mm}$. and for $\mathrm{L}$ res. $\pm 0.79 \mathrm{~mm}$. In all cases, the error was less than $1 \mathrm{~mm}$, which denoted adequate accuracy for this kind of geometry.

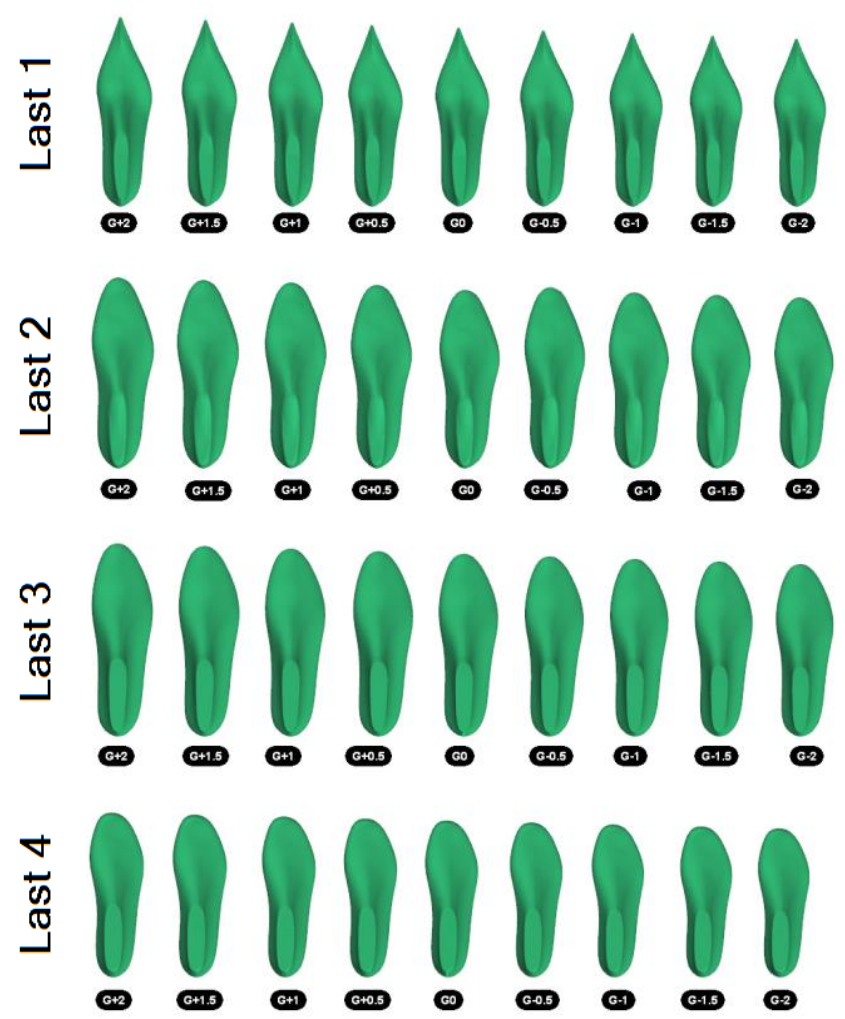

The graph in figure 11 shows, for each last, the average ball girth error as a function of the resolution. This error increased in all cases when comparing the error obtained with $\mathrm{H}$. with that obtained with $\mathrm{M}$. res., and ranged between a minimum of $0.14 \mathrm{~mm}$ (Last 1) to a maximum of $\pm 0.3 \mathrm{~mm}$ (Last 3 ). With regard to the comparison of the average error of $\mathrm{M}$ with $\mathrm{L}$, there was a decrease in two lasts and an increase in the other two, with a maximum difference of $\pm 0.25 \mathrm{~mm}$. The greatest average error difference $( \pm 0.37 \mathrm{~mm})$ between $\mathrm{H}$ and $\mathrm{L}$ was found in Last 1.

The graphs in figure 12 show the relative error with respect to the original ball girth for each of the lasts and for each size. They prove that the error was in no way related with the graded size, but with the resolution, the error obtained with $\mathrm{H}$ being less than that with $\mathrm{M}$ and $\mathrm{L}$ in $88 \%$ of the tests. The maximum error rate in $\mathrm{H}$ resolution was \pm $0.19 \%, \pm 0.57 \%$ with $\mathrm{M}$ and $\pm 0.79 \%$ with $\mathrm{L}$. In all analysed cases, the error obtained was less than $\pm 1 \mathrm{~mm}$; therefore, we can consider that the proposed method has adequate accuracy to work with meshes with a number of polygons equal to or greater than 10,000 . Nevertheless, for higher accuracy, one just has to discretise the original NURBS at a higher resolution to obtain a virtually 0 error rate (as the resolution increases, the error decreases).

Another important aspect to be analysed is the execution time as a function of the resolution. Figure 13 shows the average grading calculation time for each resolution and each last.

Fig. 10 Set of graded sizes for all lasts in the experimental stage

\begin{tabular}{|c|c|c|c|c|c|c|c|c|c|c|c|c|}
\hline & \multicolumn{3}{|c|}{ Last 1} & \multicolumn{3}{|c|}{ Last 2} & \multicolumn{3}{|c|}{ Last 3} & \multicolumn{3}{|c|}{ Last 4} \\
\hline Total length & \multicolumn{3}{|c|}{$273.33 \mathrm{~mm}$} & \multicolumn{3}{|c|}{$269.15 \mathrm{~mm}$} & \multicolumn{3}{|c|}{$247.38 \mathrm{~mm}$. } & \multicolumn{3}{|c|}{$277.36 \mathrm{~mm}}$. \\
\hline \multirow[t]{2}{*}{ Effective length } & \multicolumn{3}{|c|}{$239.76 \mathrm{~mm}$. } & \multicolumn{3}{|c|}{$246.42 \mathrm{~mm}}$. & \multicolumn{3}{|c|}{$239.76 \mathrm{~mm}}$. & \multicolumn{3}{|c|}{$266.40 \mathrm{~mm}}$. \\
\hline & Eur & UK & Mon. & Eur & UK & Mon. & Eur & UK & Mon. & Eur & UK & Mon. \\
\hline Base size & 36 & 3.5 & 235 & 37 & 4 & 245 & 36 & 3.5 & 235 & 40 & 6.5 & 265 \\
\hline Size -2 & 34 & 1.5 & 225 & 35 & 2.5 & 230 & 34 & 1.5 & 225 & 38 & 5 & 250 \\
\hline Size -1.5 & 34.5 & 2 & 225 & 35.5 & 3 & 235 & 34.5 & 2 & 225 & 38.5 & 5.5 & 255 \\
\hline Size -1 & 35 & 2.5 & 230 & 36 & 3.5 & 235 & 35 & 2.5 & 230 & 39 & 5.5 & 255 \\
\hline Size -0.5 & 35.5 & 3 & 235 & 36.5 & 3.5 & 240 & 35.5 & 3 & 235 & 39.5 & 6 & 260 \\
\hline Size 0.5 & 36.5 & 3.5 & 240 & 37.5 & 4.5 & 245 & 36.5 & 3.5 & 240 & 40.5 & 7 & 265 \\
\hline Size 1 & 37 & 4 & 245 & 38 & 5 & 250 & 37 & 4 & 245 & 41 & 7 & 270 \\
\hline Size 1.5 & 37.5 & 4.5 & 245 & 38.5 & 5.5 & 255 & 37.5 & 4.5 & 245 & 41.5 & 7.5 & 275 \\
\hline Size 2 & 38 & 5 & 250 & 39 & 5.5 & 255 & 38 & 5 & 250 & 42 & 8 & 275 \\
\hline
\end{tabular}

Table 2. Size equivalences for grading experiments. 


\begin{tabular}{|c|c|c|c|c|c|c|c|c|c|c|c|c|}
\hline & & Last 1 & & & Last 2 & & & Last 3 & & & Last 4 & \\
\hline$\Delta$ Size & H Res & M Res & L Res & H Res & M Res & L Res & H Res & M Res & L Res & H Res & M Res & L Res \\
\hline Size -2 & 0.00 & 0.00 & 0.02 & 0.00 & 0.00 & 0.01 & 0.00 & 0.01 & 0.03 & 0.00 & 0.01 & 0.05 \\
\hline Size -1.5 & 0.00 & 0.00 & 0.02 & 0.00 & 0.00 & 0.01 & 0.00 & 0.01 & 0.03 & 0.01 & 0.01 & 0.05 \\
\hline Size -1 & 0.00 & 0.00 & 0.02 & 0.00 & 0.00 & 0.01 & 0.00 & 0.01 & 0.03 & 0.01 & 0.01 & 0.05 \\
\hline Size -0.5 & 0.00 & 0.00 & 0.02 & 0.00 & 0.00 & 0.01 & 0.00 & 0.01 & 0.03 & 0.01 & 0.01 & 0.05 \\
\hline Size 0.5 & 0.00 & 0.00 & 0.02 & 0.00 & 0.00 & 0.01 & 0.00 & 0.01 & 0.04 & 0.01 & 0.01 & 0.06 \\
\hline Size 1 & 0.00 & 0.00 & 0.02 & 0.00 & 0.00 & 0.01 & 0.00 & 0.01 & 0.04 & 0.01 & 0.00 & 0.06 \\
\hline Size 1.5 & 0.00 & 0.00 & 0.02 & 0.00 & 0.00 & 0.01 & 0.00 & 0.01 & 0.04 & 0.01 & 0.00 & 0.06 \\
\hline Size 2 & 0.00 & 0.00 & 0.02 & 0.00 & 0.01 & 0.01 & 0.00 & 0.01 & 0.04 & 0.01 & 0.00 & 0.06 \\
\hline
\end{tabular}

Table 3. Effective length error

\begin{tabular}{l|ccc|ccc|ccc|ccc}
\hline & \multicolumn{3}{|c}{ Last 1 } & \multicolumn{3}{c|}{ Last 2 } & \multicolumn{3}{c}{ Last 3 } & \multicolumn{3}{c}{ Last 4 } \\
$\Delta$ Size & H Res & M Res & L Res & H Res & M Res & L Res & H Res & M Res & L Res & H Res & M Res & L Res \\
\hline Size -2 & 0.34 & 0.30 & 0.55 & 0.11 & 0.38 & 0.67 & 0.09 & 0.29 & 0.20 & 0.15 & 0.37 & 0.22 \\
\hline Size -1.5 & 0.01 & 0.30 & 0.56 & 0.24 & 0.38 & 0.68 & 0.05 & 0.33 & 0.22 & 0.11 & 0.37 & 0.23 \\
\hline Size -1 & 0.01 & 0.30 & 0.57 & 0.28 & 0.38 & 0.68 & 0.03 & 0.34 & 0.17 & 0.06 & 0.33 & 0.19 \\
\hline Size -0.5 & 0.01 & 0.24 & 0.52 & 0.29 & 0.40 & 0.69 & 0.03 & 0.33 & 0.14 & 0.02 & 0.29 & 0.14 \\
\hline Size 0.5 & 0.01 & 0.12 & 0.41 & 0.34 & 0.55 & 0.70 & 0.00 & 0.39 & 0.10 & 0.08 & 0.21 & 0.06 \\
\hline Size 1 & 0.01 & 0.00 & 0.35 & 0.39 & 0.57 & 0.70 & 0.04 & 0.40 & 0.11 & 0.08 & 0.21 & 0.10 \\
\hline Size 1.5 & 0.01 & 0.06 & 0.24 & 0.46 & 0.54 & 0.75 & 0.04 & 0.41 & 0.02 & 0.08 & 0.23 & 0.10 \\
\hline Size 2 & 0.01 & 0.13 & 0.18 & 0.42 & 0.50 & 0.79 & 0.01 & 0.36 & 0.07 & 0.09 & 0.24 & 0.10 \\
\hline
\end{tabular}

Table 4. Ball girth error

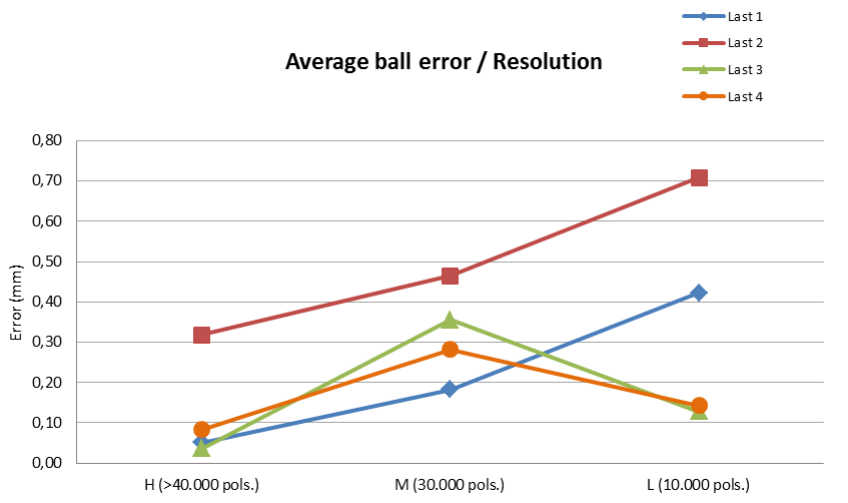

Fig. 8 Average ball girth error as a function of the resolution.
The main conclusion is that time is directly proportional to the number of polygons contained in the mesh. In the case of $\mathrm{H}$, where the resolution is not fixed, in the mesh with the greatest number of polygons (Last 3, 100,000 polygons) the time taken was 27 s., while Last 1, with 41,000 polygons, took 11 s. For M and L lasts, with a constant number of polygons, the time taken was more or less the same. In the case of M, the average time per grading was about 5 s., which means an increase of 3.3 with respect to $M$, and 7.3 with respect to the best case of $H$, and 18 times with respect to the worst case.

With a view to knowing whether the algorithm operates faster with some sizes than with others, we analysed the time taken for each size of each last, working at $\mathrm{H}$ resolution, as depicted in figure 14. No similar execution patterns were observed in the different lasts, so it was not possible to establish a relationship between the size to be graded and the speed; i.e. the resolution speed in no way depends on the selected size. 
According to the information set forth in this paper, it can be concluded that the grading speed is directly related to the resolution at which the original NURBS surface is discretised and, therefore, with the number of polygons contained in the mesh. The time taken for grading at the lowest resolution was approx. $1.5 \mathrm{~s}$., which means that it is possible to obtain a whole range of graded lasts of a given

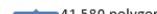

Last 1 - Ball girth error / Size

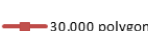

$\simeq 10,000$ polygons
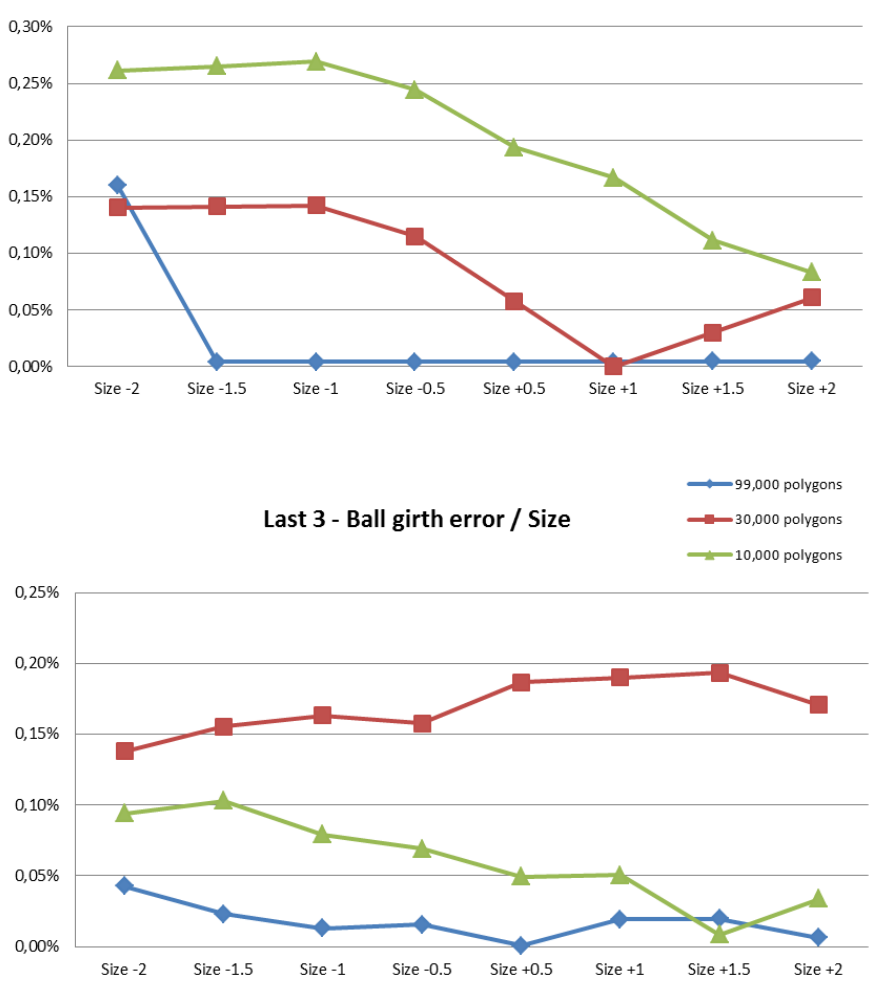

Fig. 12 Ball girth error as a function of last size

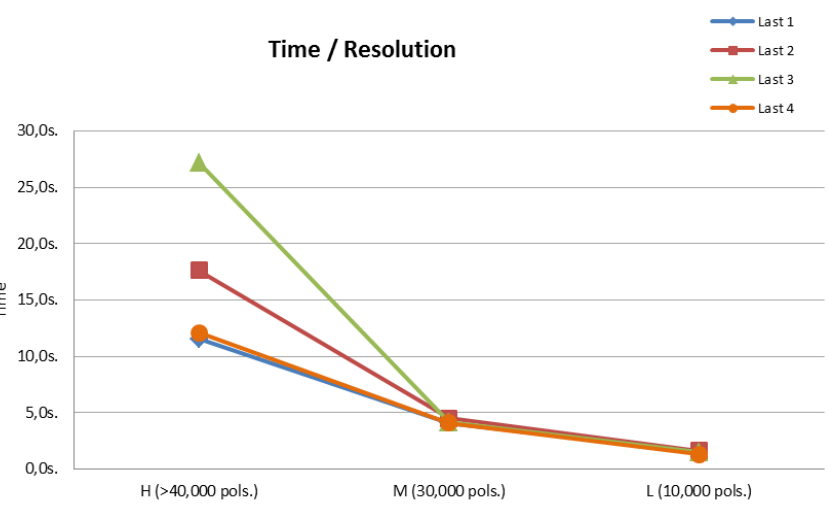

Fig. 13 Average execution times as a function of the resolution

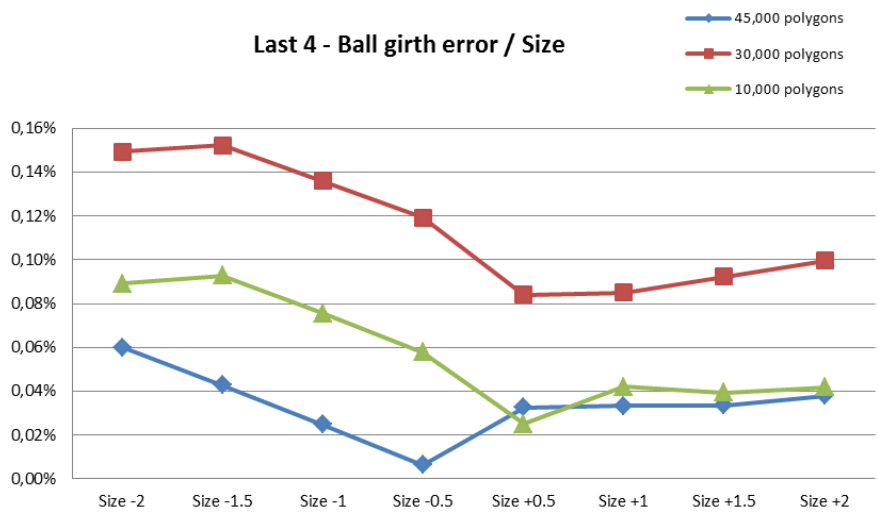

shoe model in less than $30 \mathrm{~s}$. In all cases, the error obtained was acceptable for this type of operations. Nevertheless, when working at higher accuracy, the error decreased as the discretisation resolution increased, which implied an increase in the execution time.

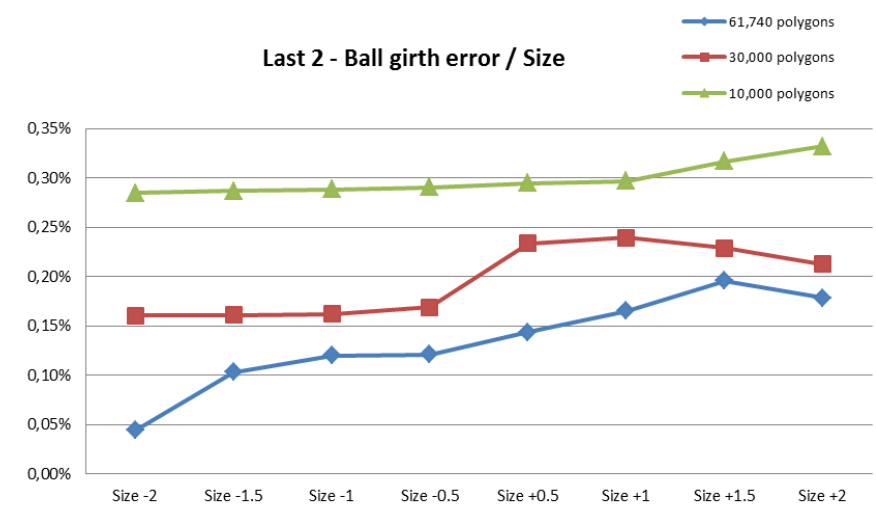

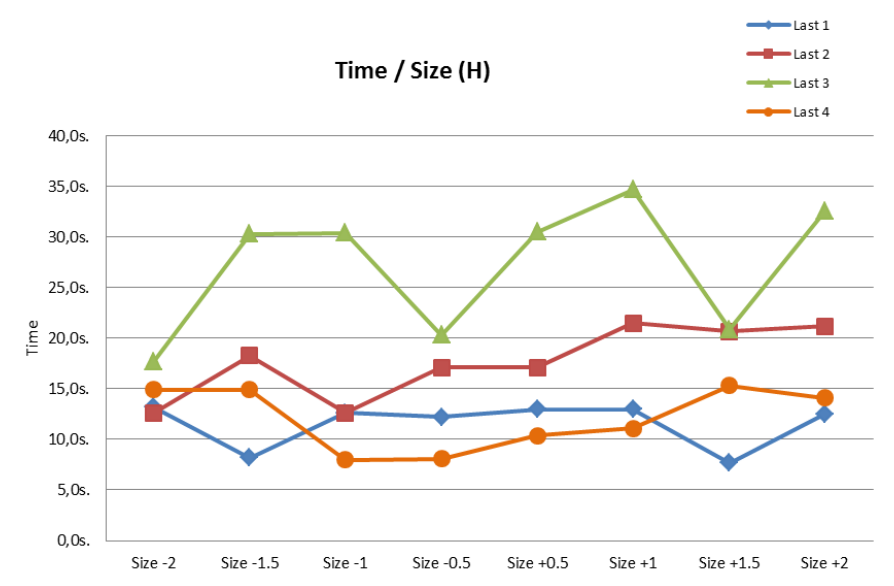

Fig. 14 Average execution times as a function of size for $\mathrm{H}$ resolution. 


\section{Conclusions}

Shoe last grading is an essential process for footwear design and manufacture, and should be accurately developed so that the resulting lasts correctly match the features of the corresponding foot size. Different CAD/CAM tools have been recently developed to carry out this process automatically. However, given the high number of grading operations that have to be performed on each last, it is important that the result is highly accurate and obtained as fast as possible. Furthermore, grading should conform to international standards relative to size marking, to enhance marketing through new channels, such as online sale, where the customer is to choose the size without the possibility of trying the shoes on.

This paper proposes a new technique to address the shoe last grading issues based on the search of the adequate grading parameters by means of the conjugate gradient method. Such parameters are obtained through the minimisation of the difference between the measures obtained and the desired ones, taking the effective length and ball girth as references. The main advantage of this method is that it allows the grading operation to be performed in a reduced time without compromising the accuracy. Besides, the proposed method complies with the international standard relative to the harmonisation of footwear sizing, so the measures obtained will be correct, not only for the footwear factory itself, but also for the global market.

The previous steps required for the application of this method do not take much time; it is just necessary to discretise the NURBS surface into a triangle mesh and position the mesh, and the designer should set the start and end points of the effective length.

According to the battery of tests carried out, we can conclude that the distinctive feature of this technique lies in the fact that the execution speed increases as the resolution of the reference discretised last decreases. While working with lower resolution meshes is a detriment to the accuracy of the algorithm, the tests carried out at a lower resolution still yielded accurate results in a very short time, about 1.5 S.

The possibility of grading lasts in a reduced time is useful for other purposes in addition to their subsequent manufacture. For future research, we propose establishing a procedure that allows carrying out exhaustive queries in lasts databases to find the last that best matches certain parameters. The lasts would be stored in their base size, and it would be necessary to grade them to the same size of the reference last in order to compare geometries of equivalent sizes. The query using low resolution meshes would be useful to carry out a high-speed initial selection and then work at a high resolution on the yielded set of lasts to obtain results with an error margin of less than 0.1 $\mathrm{mm}$.

\section{References}

[1] Wang, C. S. An analysis and evaluation of fitness for shoe lasts and human feet. Computers in Industry, vol. 61(6), pp. 532-540. (2010)

[2] Cheng, F. T., \& Perng, D. B.: A systematic approach for developing a foot size information system for shoe last design. International Journal of Industrial Ergonomics, vol. 25(2), pp. 171-185. (1999)

[3] Thompson, A. L. T.: A South African podometric study-Does the shoe fit the foot? (Doctoral dissertation). (2008)

[4] Mochimaru, M., Kouchi, M., \& Dohi, M.: Analysis of 3-D human foot forms using the free form deformation method and its application in grading shoe lasts. Ergonomics, vol. 43(9), pp. 1301-1313. (2000)

[5] Driscu, M., \& Costea, M.: Shoe last grading and experimental estimation of dimensional variation using Delcam Crispin 3D-Last Maker. Revista de pielarie incaltaminte, vol. 13(2) pp. 125-138. (2013).

[6] Kim, S. H., Shin, K. H., \& Chung, W.: A method for modifying a surface model with non-uniformly scattered displacement constraints for shoe sole design. Advances in Engineering Software, vol. 39(9), pp. 713-724. (2008)

[7] Luximon, A., Zhang, Y., Luximon, Y., \& Xiao, M. Sizing and grading for wearable products. Computer-Aided Design, vol. 44(1), pp. 77-84. (2012)

[8] Xiong J., Zhao J., Jiang Z., Dong M. A computer-aided design system for foot-feature-based shoe last customization. Int .J. Advanced Manufacturing Technoly vol. 46 pp. 11-19 (2010)

[9] Tuan, P.N., Tuan, H.M., Hien, T.T.T., Bong, T.D.: Research and Development of A New Grading Software in Footwear Industry. Science \& Technology Development, vol. 12(16). (2009).

[10] Davia, M., Jimeno-Morenilla, A., Salas, F., Footwear Biomodelling: An Industrial Approach. Computer aided design 45 (12) , pp. 1575 1590. (2013)

[11] ISO/TS 19407:2015 - Footwear Sizing: Conversion of sizing systems

[12] ISO/TS 19408:2015 - Footwear Sizing: Vocabulary and Terminology.

[13] Chen, J., Gong, Y., Jin, T., \& Tong, S. Development of an integrated CAD/CAM system for shoe last. In Mechatronics and Automation, 2005 IEEE International Conference, vol. 2, pp. 1107-1111. (2005).

[14] Jimeno, A., \& Cuenca, S. Reconfigurable computing for tool-path computation. The International Journal of Advanced Manufacturing Technology, 21(12), pp. 945-951. (2003). 